\title{
PARTITIONS INTO DISTINCT LARGE PARTS
}

\author{
GREGORY A. FREIMAN and JANE PITMAN
}

(Received 15 June 1992)

Communicated by J. H. Loxton

\begin{abstract}
An asymptotic estimate is obtained for the number of partitions of the positive integer $n$ into distinct parts, each of which is at least $m$. The estimate holds uniformly with respect to positive $m$ such that $m=o\left(n(\log n)^{-9}\right)$, as $n \rightarrow \infty$.
\end{abstract}

1991 Mathematics subject classification (Amer. Math. Soc.): 11P68, $11 \mathrm{P} 72$.

\section{Introduction}

We define $q_{m}(n)$ as the number of partitions of the positive integer $n$ into distinct parts, each of which is at least $m$, that is, the number of partitions of the type

$$
\begin{gathered}
n=i_{1}+i_{2}+\cdots+i_{r}, \\
m \leq i_{1}<i_{2}<\cdots<i_{r} \leq n .
\end{gathered}
$$

Our aim here is to obtain an asymptotic estimate of $q_{m}(n)$ as $n \rightarrow \infty$, valid for as much as possible of the range $1 \leq m \leq n / 2$. The estimate obtained will in fact be valid uniformly with respect to positive $m$ such that

$$
m=o\left(n(\log n)^{-9}\right) .
$$

The asymptotic behaviour of $q(n)=q_{1}(n)$, the number of partitions of $n$ into distinct parts, was sketched by Hardy and Ramanujan at the end of their study [8] of the classical partition function $p(n)$, and $q(n)$ was further investigated by Hua [9]. In particular, the estimate

$$
q(n) \sim \frac{1}{2^{2} 3^{1 / 4} n^{3 / 4}} \exp \left\{\pi\left(\frac{n}{3}\right)^{1 / 2}\right\}
$$

(C) 1994 Australian Mathematical Society 0263-6115/94\$A2.00+0.00 
follows from the more precise expansion in [8] and [9]. (See also Freiman [6]).

The work of Hardy and Ramanujan [8] led to extensive investigation by many authors of the asymptotic behaviour of a variety of functions arising in the theory of partitions. (See, for example, Roth and Szekeres [11]).

Over the past few years Erdös, Nicolas and Szalay have investigated the asymptotic behaviour of $q_{m}(n)$ (see [4] and [3]) and Dixmier and Nicolas have considered the related function $p_{m}(n)$, the number of partitions of $n$ into parts each of which is at least $m$ (see, for example, [2]). These studies have concentrated on the case when $m$ is relatively small (less than $n^{1 / 2}$ ). For small $m, q_{m}(n)$ is reasonably comparable with $q(n)$ and $p_{m}(n)$ with $p(n)$.

The method used here is different from the methods in the papers cited above and relies on a probabilistic approach. We represent $q_{m}(n)$ via an integral and interpret this integral in terms of a sum of independent random variables. If an appropriate local limit theorem were available, it would yield an asymptotic estimate for $q_{m}(n)$. By exploiting the special features of this particular problem we obtain, in effect, the equivalent of a local limit theorem in this situation, and this yields the required estimate.

Applications of local limit theorems to number theory were used, for example, by Khinchin and Postnikov (see Postnikov [10]). The idea of deriving the equivalent of a local limit theorem for the purposes of a particular number theoretic problem has been used previously by Freiman $[5,6,7]$.

In Section 2 we present the probabilistic considerations relevant to our problem and these lead to the formulation of our main result, Theorem 1, giving an estimate for $q_{m}(n)$ which yields an asymptotic estimate which is valid for $m$ subject to (1.1). In Sections 3, 4 and 5 we derive the various estimates and bounds needed for the proof of the theorem, and in Section 6 we complete the proof.

The main term of the estimate for $q_{m}(n)$ in Theorem 1 involves a parameter $\sigma$ which is not given explicitly in terms of $m$ and $n$. In order to give the main term explicitly in terms of $m$ and $n$ we obtain some further more precise estimates in Section 7. Then (Theorem 2) in Section 8 we derive an explicit asymptotic estimate for $q_{m}(n)$ which is valid for large $m$ and involves the inverse of the strictly decreasing function $F$ defined by

$$
F(X)=\frac{1}{X^{2}} \int_{X}^{\infty} \frac{y}{1+e^{y}} d y \quad(X>0) .
$$

As a corollary, we obtain a family of asymptotic estimates for $q_{m}(n)$ in terms of elementary functions, each estimate being valid for large $m$ in a specified interval of length at least $o\left(n^{1 / 3}\right)$.

Finally (Theorem 3), in Section 9, we derive an explicit asymptotic estimate for $q_{m}(n)$ for small $m$ which is valid for $m=o\left(n^{1 / 3}\right)$ and involves only elementary functions. The case $m=1$ yields (1.2), and the relationship to the results of [3] is 
discussed further at the end of Section 9.

We are grateful for helpful comments from participants in the Journeees Additives held at Marseille in September 1991. In particular, we thank Professor Nicolas for sending the information about the expansion of $F^{-1}(Y)$ which is discussed at the end of Section 8 .

\section{Probabilistic motivation}

Our starting point is the following lemma.

LEMMA 1. For any real $\sigma$,

$$
q_{m}(n)=e^{\sigma n} \int_{0}^{1} \prod_{j=m}^{n}\left(1+e^{-j \sigma} e^{2 \pi i \alpha j}\right) e^{-2 \pi i \alpha n} d \alpha .
$$

PROOF. The integral on the right-hand side is

$$
\sum_{\mathbf{x}} \int_{0}^{1} e^{-\sigma\left(m x_{m}+\cdots+n x_{n}\right)} e^{2 \pi i\left(m x_{m}+\cdots+n x_{n}-n\right) \alpha} d \alpha,
$$

where $\mathbf{x}$ runs over all $(n-m+1)$-tuples $\left(x_{m}, \ldots, x_{n}\right)$ such that $x_{i} \in\{0,1\}$ for all $i$. The contribution to this sum from $\mathbf{x}$ is $e^{-\sigma n}$ if

$$
m x_{m}+\cdots+n x_{n}=n
$$

and zero otherwise. Since there is an obvious one-to-one correspondence between partitions of the required type and vectors $\mathbf{x}$ such that (2.1) holds and $x_{i} \in\{0,1\}$ for all $i$, the above sum must be $e^{-\alpha n} q_{m}(n)$, giving the result.

We rewrite our expression for $q_{m}(n)$ as

$$
q_{m}(n)=e^{\sigma n} \prod_{j=m}^{n}\left(1+e^{-\sigma j}\right) \int_{0}^{1} \varphi(\alpha) e^{-2 \pi i \alpha n} d \alpha,
$$

where

$$
\begin{gathered}
\varphi(\alpha)=\prod_{j=m}^{n} \varphi_{j}(\alpha), \\
\varphi_{j}(\alpha)=p_{1 j}+p_{2 j} e^{2 \pi i \alpha j}, \\
p_{1 j}=\frac{1}{1+e^{-\sigma j}}, \quad p_{2 j}=\frac{e^{-\sigma j}}{1+e^{-\sigma j}}=\frac{1}{1+e^{\sigma j}} \quad(m \leq j \leq n) .
\end{gathered}
$$


Consider independent variables $X_{m}, X_{m+1}, \ldots, X_{n}$ such that

$$
X_{j}= \begin{cases}0 & \text { with probability } p_{1 j}, \\ j & \text { with probability } p_{2 j}\end{cases}
$$

so that $X_{j}$ has characteristic function $\varphi_{j}$ given by (2.4) above. Then $\varphi$ in (2.3) is the characteristic function of

$$
Y=X_{m}+X_{m+1}+\cdots+X_{n}
$$

We define

$$
M=\sum_{j=m}^{n} \frac{j}{1+e^{\sigma j}}, \quad B^{2}=\sum_{j=m}^{n} \frac{j^{2} e^{\sigma j}}{\left(1+e^{\sigma j}\right)^{2}} .
$$

Then $Y$ has mean $E(Y)$ and variance $V(Y)$ given by

$$
E(Y)=M, \quad V(Y)=B^{2} .
$$

If a local limit theorem applied in this situation we would have

$$
P(Y=n) \sim\left(2 \pi B^{2}\right)^{-1 / 2} e^{-(M-n)^{2} /\left(2 B^{2}\right)} \quad \text { as } n \rightarrow \infty .
$$

We define the parameter $\sigma=\sigma(m, n)$ as the unique real number such that

$$
n=\sum_{j=m}^{n} \frac{j}{1+e^{\sigma j}},
$$

so that $E(Y)=M=n$. Now

$$
P(Y=n)=\int_{0}^{1} \varphi(\alpha) e^{-2 \pi i \alpha n} d \alpha,
$$

and hence with the above choice of $\sigma$ our limit theorem would yield

$$
\int_{0}^{1} \varphi(\alpha) e^{-2 \pi i \alpha n} d \alpha \sim\left(2 \pi B^{2}\right)^{-1 / 2}
$$

Combination of this with (2.2) leads to the conjecture that, as $n \rightarrow \infty$,

$$
q_{m}(n) \sim\left(2 \pi B^{2}\right)^{-1 / 2} e^{\sigma n} \prod_{j=m}^{n}\left(1+e^{-j \sigma}\right),
$$

under suitable conditions on $m$. We can now formulate our main result, which is the following more precise version of this conjecture. 
THEOREM 1. As $n \rightarrow \infty$ we have

$$
q_{m}(n)=\left(2 \pi B^{2}\right)^{-1 / 2} e^{\sigma n} \prod_{j=m}^{n}\left(1+e^{-j \sigma}\right)(1+E),
$$

where $B$ and $\sigma$ are given by (2.6) and (2.7) and

$$
E=E(m, n)=O\left((\log n)^{9 / 2} \max \left\{\frac{1}{n^{1 / 4}},\left(\frac{m}{n}\right)^{1 / 2}\right\}\right)
$$

uniformly with respect to $m$ such that

$$
1 \leq m \leq K_{0} n(\log n)^{-9} .
$$

Here $K_{0}$ and the implied constants in the estimate of $E$ are effective positive constants independent of $m$ and $n$.

By taking $m$ such that $1 \leq m \leq K_{0} n(\log n)^{-9} \theta_{n}$, where $\left(\theta_{n}\right)$ is a non-negative decreasing sequence such that $\theta_{n} \rightarrow 0$ as $n \rightarrow \infty$, we see that, as $n \rightarrow \infty, E(m, n)$ tends to zero uniformly with respect to $m$ in the given range. Thus we obtain

COROLlaRY. As $n \rightarrow \infty$ we have

$$
q_{m}(n) \sim\left(2 \pi B^{2}\right)^{-1 / 2} e^{\sigma n} \prod_{j=m}^{n}\left(1+e^{-j \sigma}\right),
$$

uniformly with respect to positive $m$ such that $m=o\left(n(\log n)^{-9}\right)$.

In order to prove Theorem 1 we need to prove that for $m$ and $n$ as in (2.9)

$$
\int_{0}^{1} \varphi(\alpha) e^{-2 \pi i \alpha n} d \alpha=\left(2 \pi B^{2}\right)^{-1 / 2}(1+E)
$$

with $E$ as in the theorem. Let

$$
J=\int_{-\frac{1}{2}}^{\frac{1}{2}} \varphi(\alpha) e^{-2 \pi i \alpha n} d \alpha,
$$

so that, by periodicity, $J$ coincides with the given integral. To prove the theorem it will be sufficient to show that $J=\left(2 \pi B^{2}\right)^{-1 / 2}(1+E)$ with a suitable upper bound on $|E|$. The aim of our preliminary work will be to provide a basis for proving that as $n \rightarrow \infty$ we have

$$
J \sim\left(2 \pi B^{2}\right)^{-1 / 2} .
$$




\section{Estimation of the integrand and the main term}

We shall need the fact that $\sigma>0$ and we therefore start with preliminary estimates of $\sigma$.

LEMMA 2. For $\sigma$ as in (2.7) and $1 \leq m \leq n / 2$ we have the following estimates.

(i) If $n \geq 6$ then $0<\sigma<2 / \sqrt{ } n$.

(ii) Let $r$ be an integer such that

$$
\max \{m, 2 \sqrt{n}\} \leq r \leq n / 2 .
$$

Then

$$
\sigma>\frac{1}{r} \log \left(\frac{r}{2 \sqrt{ } n}\right) .
$$

PROOF. (i) Suppose $n \geq 6$ and $1 \leq m \leq n / 2$. If $\sigma \leq 0$ then $e^{\sigma j} \leq 1$ for each $j$ and so by (2.7) we have

$$
\begin{aligned}
n \geq \frac{1}{2} \sum_{j=m}^{n} j & =(n+m)(n-m+1) / 4 \\
> & \left(n^{2}-m^{2}\right) / 4 \geq 3 n^{2} / 16,
\end{aligned}
$$

which implies $n<6$, a contradiction. Thus $\sigma>0$.

Write $q=e^{-\sigma}$, where $0<q<1$ since $\sigma>0$. By (2.7) we now have

$$
n<\sum_{j=m}^{n} j q^{j}<\sum_{j=1}^{\infty} j q^{j}=q(1-q)^{-2}<(1-q)^{-2} .
$$

It follows that

$$
e^{\sigma}=\frac{1}{q}<\frac{\sqrt{ } n}{\sqrt{ } n-1}<1+\frac{2}{\sqrt{ } n} .
$$

Since $\sigma<e^{\sigma}-1$, the given upper bound for $\sigma$ now follows.

(ii) Suppose (3.1) holds. Then by (2.7) and the positiveness of $\sigma$ we have

$$
n>\sum_{j=r}^{2 r} \frac{j}{e^{\sigma j}+1}>\sum_{j=r}^{2 r} \frac{j}{2 e^{2 \sigma r}}>\frac{r^{2}}{4 e^{2 \sigma r}} .
$$

Hence

$$
2 \sigma r>\log \left(\frac{r^{2}}{4 n}\right)=2 \log \left(\frac{r}{2 \sqrt{ } n}\right),
$$

which gives the required lower bound for $\sigma$.

Now we give estimates of $\varphi_{j}(\alpha)$. 
LEMMA 3. For $\varphi_{j}(\alpha)$ as in (2.4) we have the following estimates.

(i) For all real $\alpha$,

$$
\left|\varphi_{j}(\alpha)\right| \leq \exp \left(-2 p_{1 j} p_{2 j} \sin ^{2} \pi \alpha j\right) .
$$

(ii) If

$$
p_{2 j}|\sin \pi \alpha j| \leq 1 / 3
$$

then

$$
\varphi_{j}(\alpha)=\exp \left(2 \pi i \alpha j p_{2 j}-2 \pi^{2} \alpha^{2} j^{2} p_{1 j} p_{2 j}+O\left(\alpha^{3} j^{3} p_{2 j}\right)\right),
$$

with the implied constant effective and independent of $\alpha, m, n$ and $j$.

ProOF. (i) We have by (2.4) and (2.5) and the fact that $p_{1 j}+p_{2 j}=1$,

$$
\begin{aligned}
\left|\varphi_{j}(\alpha)\right|^{2} & =p_{1 j}^{2}+p_{2 j}^{2}+2 p_{1 j} p_{2 j} \cos 2 \pi \alpha j \\
& =1-2 p_{1 j} p_{2 j}(1-\cos 2 \pi \alpha j) \\
& =1-4 p_{1 j} p_{2 j} \sin ^{2} \pi \alpha j \\
& \leq \exp \left(-4 p_{1 j} p_{2 j} \sin ^{2} \pi \alpha j\right),
\end{aligned}
$$

since $1-\theta \leq e^{-\theta}$ if $\theta \geq 0$.

(ii) We can rewrite (2.4) as

$$
\varphi_{j}(\alpha)=1+p_{2 j}\left(e^{2 \pi i \alpha j}-1\right)=1+\theta,
$$

say, where

$$
\begin{aligned}
\theta=p_{2 j}\left(e^{2 \pi i \alpha j}-1\right) & =p_{2 j}(\cos 2 \pi \alpha j+i \sin 2 \pi \alpha j-1), \\
|\theta| & =2 p_{2 j}|\sin \pi \alpha j| .
\end{aligned}
$$

Since $|\theta| \leq 2 / 3$ by (3.2), it follows from the series expansion for $\log (1+\theta)$ that

$$
1+\theta=\exp \left(\theta-\theta^{2} / 2+O\left(\theta^{3}\right)\right),
$$

with implied constant effective and independent of all our parameters. By expressing $\theta, \theta^{2}, \theta^{3}$ in terms of cosines and sines of multiples of $2 \pi \alpha j$ and then applying Taylor's theorem to $\cos 2 \pi \alpha j, \sin 2 \pi \alpha j, \cos 4 \pi \alpha j$, etcetera, (cf. Breiman [1, Proof of Prop. 8.44]) we obtain

$$
\begin{aligned}
\theta & =p_{2 j}\left(2 \pi i \alpha j-(2 \pi \alpha j)^{2} / 2+O(\alpha j)^{3}\right), \\
\theta^{2} & =p_{2 j}^{2}\left(-(2 \pi \alpha j)^{2}+O(\alpha j)^{3}\right), \\
\theta^{3} & =p_{2 j}^{3} O(\alpha j)^{3} .
\end{aligned}
$$

The required estimate follows from these results and (3.3), since

$$
0<p_{2 j}<1, \quad p_{2 j}-p_{2 j}^{2}=p_{1 j} p_{2 j} .
$$


The above lemma enables us to estimate $\varphi(\alpha)$.

COROLLARY. We have the following estimates for $\varphi(\alpha)$ as in (2.3).

(i) For all real $\alpha,|\varphi(\alpha)| \leq \exp \left(-\sum_{j=m}^{n} 2 p_{1 j} p_{2 j} \sin ^{2} \pi \alpha j\right)$.

(ii) Suppose that either $m \sigma \geq 1$ or $|\alpha| \leq \sigma /(3 \pi)$. Then

$$
\varphi(\alpha)=e^{2 \pi i n \alpha} \exp \left(-2 \pi^{2} \alpha^{2} B^{2}+O\left(\alpha^{3} \rho_{3}\right)\right),
$$

where $n, \sigma$ and $B$ satisfy (2.7) and (2.6),

$$
\rho_{3}=\sum_{j=m}^{n} \frac{j^{3}}{1+e^{\sigma j}},
$$

and the implied constant is effective and independent of $m, n$ and $\alpha$.

PROOF. The inequality of (i) follows by multiplying the estimates for $\left|\varphi_{j}(\alpha)\right|$ for $j=m, m+1, \ldots, n$ given by part (i) of the Lemma. For (ii), we note that if $\sigma j \geq 1$ then by (2.5) $p_{2 j}<(1+e)^{-1}<1 / 3$, while if $\sigma j<1$ then $j<\sigma^{-1}$ and so $|\sin \pi \alpha j| \leq|\pi \alpha j|<|\pi \alpha| / \sigma$. Thus our hypotheses on $m$ and $\alpha$ ensure that (3.2) holds and the estimate given by part (ii) of the lemma applies for $j=m, m+1, \ldots, n$. Combining these estimates and using (2.7), we obtain the required result.

Let $\alpha_{0}$ be a positive parameter such that $0<\alpha_{0}<1 / 2$ (to be specified more precisely later on). The above corollary now enables us to estimate the contribution to $J$ as in (2.10) from the subset $\left[-\alpha_{0}, \alpha_{0}\right]$ and suggests that, for suitable positive $\alpha_{0}$, this may dominate the contribution from the remainder of $[-1 / 2,1 / 2]$, which will be called the supplementary subset.

LEMMA 4. Let

$$
J_{0}=J_{0}\left(n, \alpha_{0}\right)=\int_{-\alpha_{0}}^{\alpha_{0}} e^{-2 \pi i n \alpha} \varphi(\alpha) d \alpha,
$$

where

$$
(2 \pi B)^{-1} \leq \alpha_{0} \leq \min \left(\sigma(3 \pi)^{-1},\left(\rho_{3}\right)^{-1 / 3}\right),
$$

with $B, \sigma$ and $\rho_{3}$ defined by (2.6), (2.7) and (3.4).

Then

$$
J_{0}=\left(2 \pi B^{2}\right)^{-1 / 2}\left(1+O\left(e^{-2 \pi^{2} \alpha_{0}^{2} B^{2}}\right)+O\left(\alpha_{0}^{3} \rho_{3}\right)\right),
$$

where $\rho_{3}$ is given by (3.4) and the implied constants are effective and independent of $m, n$ and $\alpha_{0}$.

PROOF. It follows from (ii) of the above corollary and the boundedness of $\alpha_{0}^{3} \rho_{3}$ that

$$
J_{0}=\int_{-\alpha_{0}}^{\alpha_{0}} \exp \left(-2 \pi^{2} \alpha^{2} B^{2}\right) d \alpha\left(1+O\left(\alpha_{0}^{3} \rho_{3}\right)\right)
$$


Now (putting $2 \pi \alpha B=y$ )

$$
\int_{-\alpha_{0}}^{\alpha_{0}} \exp \left(-2 \pi^{2} \alpha^{2} B^{2}\right) d \alpha=\frac{1}{2 \pi B}\left(\int_{-\infty}^{\infty} e^{-y^{2} / 2} d y+O\left(\int_{2 \pi \alpha_{0} B}^{\infty} e^{-y^{2} / 2} d y\right)\right) .
$$

The first integral is $\sqrt{2 \pi}$ and since $2 \pi \alpha_{0} B \geq 1$ we have

$$
\int_{2 \pi \alpha_{0} B}^{\infty} e^{-y^{2} / 2} d y \leq \int_{2 \pi \alpha_{0} B}^{\infty} y e^{-y^{2} / 2} d y=e^{-2 \pi^{2} \alpha_{0}^{2} B^{2}} .
$$

Thus we obtain

$$
\int_{-\alpha_{0}}^{\alpha_{0}} \exp \left(-2 \pi \alpha^{2} B^{2}\right) d \alpha=\left(2 \pi B^{2}\right)^{-1 / 2}\left(1+O\left(e^{-2 \pi^{2} \alpha_{0}^{2} B^{2}}\right)\right),
$$

which gives the required result. (We shall see in Section 6 that the conditions on $\alpha_{0}$ are consistent for large enough $n$.)

If $\alpha_{0}=\alpha_{0}(n)$ satisfies

$$
\alpha_{0}^{2} B^{2} \rightarrow \infty, \quad \alpha_{0}^{3} \rho_{3} \rightarrow 0
$$

as $n \rightarrow \infty$, then Lemma 4 shows that

$$
J_{0} \sim\left(2 \pi B^{2}\right)^{-1 / 2} .
$$

If, further, the contribution to $J$ from the supplementary subset is $o\left(B^{-1}\right)$ then we shall obtain (2.11). In order to estimate the contribution from the supplementary subset and then to choose $\alpha_{0}$ appropriately, we shall clearly need careful estimates of the parameters $\sigma, \boldsymbol{B}, \rho_{3}$.

\section{Estimation of key parameters}

In this section we give preliminary estimates of the key parameters $\sigma, B$ and $\rho_{3}$ which are sufficient for proving Theorem 1 . We start by estimating sums of the type

$$
\sum_{j=r}^{s} j^{k} e^{-\sigma j}
$$

since such sums are involved in the estimation of $\sigma, B$ and $\rho_{3}$.

LEMMA 5. For $n \geq 6$, positive integral $k$ and $\sigma$ as in (2.7), we have the following estimates for sums of the type (4.1). 
(i) We have

$$
\sum_{j=1}^{n} j^{k} e^{-\sigma j} \leq C_{0} \sigma^{-k-1} \quad \text { provided } n \geq n_{0}
$$

where $C_{0}$ and $n_{0}$ are effective constants, independent of $m$ and $n$ (but dependent on $k$ ).

(ii) Let $r$ be a positive integer such that

$$
k \sigma^{-1}<r \leq n / 2
$$

Then

$$
\sum_{j=r}^{n} j^{k} e^{-\sigma j}=\left(\sigma^{-1} r^{k} e^{-\sigma r}\right)\left(1+O\left((\sigma r)^{-1}\right)\right),
$$

with the implied constant effective and independent of $m, n$ and $r$ (but dependent on $k$ ).

PROOF. (i) We consider the function $f$ defined by

$$
f(x)=(\sigma x)^{k} e^{-\sigma x}
$$

and recall that $\sigma>0$. We note that $f$ is strictly increasing on $\left[0, k \sigma^{-1}\right]$, strictly decreasing on $\left[k \sigma^{-1}, \infty\right)$, and hence has a unique maximum on $[0, \infty)$, namely

$$
f\left(k \sigma^{-1}\right)=k^{k} e^{-k}
$$

We also note that by induction on $k$ and integration by parts, we have

$$
\int_{b}^{\infty} y^{k} e^{-y} d y=e^{-b}\left(b^{k}+k b^{k-1}+k(k-1) b^{k-2}+\ldots+k !\right) .
$$

It follows by using the change of variable $y=\sigma x$ that

$$
\int_{0}^{\infty} f(x) d x=k ! \sigma^{-1}
$$

and also that

$$
\int_{a}^{\infty} f(x) d x=\sigma^{-1} f(a)\left(1+O\left((a \sigma)^{-1}\right)\right) \quad \text { if } a \sigma>1 .
$$

By applying Euler's summation formula to $f(x)$ on $\left[0, k \sigma^{-1}\right]$ and on $\left[k \sigma^{-1}, n\right]$ and using the monotonicity of $f$ on these intervals we see that

$$
\sum_{j=1}^{n} j^{k} e^{-\sigma j}=\frac{1}{\sigma^{k}} \sum_{j=1}^{n} f(j)=\frac{1}{\sigma^{k}}\left(\int_{0}^{n} f(x) d x+O\left(f\left(k \sigma^{-1}\right)\right)\right) .
$$


Since $0<\sigma<1$ by Lemma 2, the result (i) now follows from (4.3) and (4.4).

(ii) Suppose now that (4.2) holds and hence $r \sigma>1$. By applying the integral test to $f(x)$ on $[r, n]$ and using (4.5), we obtain

$$
\begin{aligned}
\sum_{j=r}^{n} j^{k} e^{-\sigma j} & =\frac{1}{\sigma^{k}}\left(\int_{r}^{n} f(x) d x+O(f(r))\right) \\
& =\frac{1}{\sigma^{k}}\left(\sigma^{-1}(f(r)-f(n))\left(1+O\left((\sigma r)^{-1}\right)\right)+O(f(r))\right)
\end{aligned}
$$

Since $n \geq 2 r$, we have

$$
f(n) \leq f(2 r)=f(r) O\left(e^{-\sigma r}\right)=f(r) O\left((\sigma r)^{-1}\right),
$$

and hence the right-hand side of (4.6) is

$$
\frac{1}{\sigma^{k+1}} f(r)\left(1+O\left((\sigma r)^{-1}\right)+O(\sigma)\right)
$$

which gives the required estimate for the above sum since $\sigma=O\left((\sigma r)^{-1}\right)$ by Lemma 2(i).

We can now give our basic estimates of $\sigma, B$ and $\rho_{3}$.

LEMMA 6. For $n \geq 6$, we have the following estimates involving $\sigma, B$ and $\rho_{3}$ as defined in (2.7), (2.6) and (3.4).

(i) For $1 \leq m \leq n / 2$, we have

$$
\begin{array}{rlr}
n & \leq 4 \sigma^{-2}, & \\
B^{2} & \leq C_{1} \sigma^{-3} & \text { provided } n \geq n_{0}, \\
\rho_{3} & \leq C_{2} \sigma^{-4} \quad & \text { provided } n \geq n_{0},
\end{array}
$$

where $C_{1}, C_{2}$ and $n_{0}$ are effective positive constants, independent of $m$ and $n$.

(ii) If

$$
3 \sigma^{-1}<m \leq n / 2
$$

then

$$
\begin{aligned}
n & =m \sigma^{-1} e^{-\sigma m}\left(1+O\left((\sigma m)^{-1}\right)\right), \\
B^{2} & =m^{2} \sigma^{-1} e^{-\sigma m}\left(1+O\left((\sigma m)^{-1}\right)\right), \\
\rho_{3} & =m^{3} \sigma^{-1} e^{-\sigma m}\left(1+O\left((\sigma m)^{-1}\right)\right),
\end{aligned}
$$

with the implied constants effective and independent of $m$ and $n$. 
PROOF. Suppose $n \geq 6$ and $1 \leq m \leq n / 2$. The upper bound for $n$ in (i) is immediate from Lemma 2 . We indicate the method for the other estimates by considering $B^{2}$ in detail. Since $\sigma>0$ it follows from (2.6) that $B^{2} \leq \sum_{j=m}^{n} j^{2} e^{-\sigma j}$, so that Lemma 5(i) with $k=2$ gives $B^{2} \leq C_{0} \sigma^{-3}$ provided $n \geq n_{0}$.

For any integer $r$ such that (4.2) holds with $k=2$, we have by Lemma 5(ii)

$$
\begin{aligned}
\sum_{j=r}^{n} \frac{j^{2} e^{\sigma j}}{\left(1+e^{\sigma j}\right)^{2}} & =\left(\sum_{j=r}^{n} j^{2} e^{-\sigma j}\right)\left(1+O\left(e^{-\sigma r}\right)\right) \\
& =\frac{1}{\sigma} r^{2} e^{-r \sigma}\left(1+O\left((\sigma r)^{-1}\right)\right) .
\end{aligned}
$$

If $m>3 \sigma^{-1}$, we apply this result with $r=m$ and obtain the estimate for $B^{2}$ in (ii).

The remaining estimates for $\rho_{3}$ and $n$ are obtained similarly by using Lemma 5 with $k=3$ and $k=1$, respectively.

We now use Lemmas 5 and 6 to obtain estimates which together cover the whole range $1 \leq m \leq n / 2$.

LEMMA 7. For $n \geq 6$, we have the following estimates involving $\sigma, B$ and $\rho_{3}$ as defined in (2.7), (2.6) and (3.4).

(i) There exists an effective constant $K \geq 3$, independent of $m$ and $n$, such that if $K \sigma^{-1}<m \leq n / 2$ then

$$
\begin{aligned}
n / 2 & \leq m \sigma^{-1} e^{-\sigma m} \leq 2 n, \\
B^{2} / 2 & \leq m^{2} \sigma^{-1} e^{-\sigma m} \leq 2 B^{2}, \\
\rho_{3} / 2 & \leq m^{3} \sigma^{-1} e^{-\sigma m} \leq 2 \rho_{3}, \\
m \sigma & \leq \log n .
\end{aligned}
$$

(ii) For $K$ as in (i) there exist constants $C=C(K) \geq 1$ and $n_{1}=n_{1}(K)$ such that if $1 \leq m \leq K \sigma^{-1}$ then

$$
\begin{array}{rlrl}
n & \geq C \sigma^{-2} & & \text { provided } n \geq n_{1}, \\
B^{2} & \geq C \sigma^{-3} & \text { provided } n \geq n_{1}, \\
\rho_{3} \geq C \sigma^{-4} & \text { provided } n \geq n_{1} .
\end{array}
$$

PROOF. (i) We choose $K \geq 3$ such that if $\sigma m>K$ then the three error terms occurring in Lemma 6(ii) all have absolute value at most $1 / 2$. This yields the first three inequalities. If $K \sigma^{-1}<m \leq n / 2$, we have, in particular $n / 2 \leq m \sigma^{-1} e^{-\sigma m}$, and hence $n \sigma e^{\sigma m} \leq 2 m \leq n$; since $n \sigma>m \sigma>1$, it then follows that $e^{\sigma m} \leq n$ and hence $\sigma m \leq \log n$. 
(ii) We now assume that $1 \leq m \leq K \sigma^{-1}$, for $K$ as in (i). We note that by Lemma 5(ii) and (4.7) there is a constant $K_{1}$ such that if $r$ is an integer such that (4.2) holds with $k=2$ and if $r \sigma \geq K_{1}$ then

$$
\sum_{j=r}^{n} \frac{j^{2}}{\left(1+e^{\sigma j}\right)^{2}} \geq \frac{1}{2 \sigma} r^{2} e^{-\sigma r} .
$$

We take

$$
A=\max \left(K, K_{1}\right),
$$

and note that by Lemma 2(ii) (with $r=[n / 2]$, for example), there exists $n_{1}=n_{1}(A)$ such that

$$
\sigma n>4 A \quad \text { if } n \geq n_{1} \text {. }
$$

We take

$$
r=\left[A \sigma^{-1}\right]+1,
$$

and note that if $n \geq n_{1}$ then (since $\sigma^{-1} \geq 1$ by Lemma 2(i) and $A \geq 2$ )

$$
m \leq A \sigma^{-1}<r \leq 2 A \sigma^{-1} \leq n / 2,
$$

so that $r$ satisfies (4.2) with $k=2$, and $r \sigma \geq K_{1}$ and $r>m$. Hence if $n \geq n_{1}$ we obtain $B^{2} \geq r^{2} e^{-\sigma r} /(2 \sigma)$ and hence, by (4.8),

$$
B^{2} \geq \frac{A^{2}}{2 \sigma^{3}} e^{-2 A}=\frac{C}{\sigma^{3}},
$$

say, where $C=C(A)$ is independent of $m$ and $n$.

The argument is easily expanded to cover the other two inequalities at the same time, by using Lemma 5 with $k=1$ and $k=3$.

COROLLARY. There exist effective positive constants $C, D, n_{0}$, independent of $m$ and $n$, such that if $1 \leq m \leq n / 2$ then $C B^{4} \leq \rho_{3} n \leq D B^{4}$ provided $n \geq n_{0}$.

PROOF. Let $K$ be the constant in Lemma 7. The result for $K \sigma^{-1}<m \leq n / 2$ follows from Lemma 7(i) and the result for $1 \leq m \leq K \sigma^{-1}$ follows from Lemma 6(i) and Lemma 7(ii). Combining these results gives the required conclusion.

\section{Contribution from the supplementary set}

The main term of our estimate of $J$ as in (2.10) will come from $J_{0}$, the contribution to $J$ from the subset $\left[-\alpha_{0}, \alpha_{0}\right]$, as estimated by Lemma 4 , for suitable positive $\alpha_{0}$ to 
be determined later (in Section 6). We shall call $\left[-\alpha_{0}, \alpha_{0}\right]$ the main subset. We now consider the contribution to $J$ as in (2.10) from the supplementary subset

$$
S=\left\{\alpha: \alpha_{0} \leq|\alpha| \leq 1 / 2\right\} .
$$

We need to show that, for suitably chosen $\alpha_{0}$, this contribution is $o\left(B^{-1}\right)$ as $n \rightarrow \infty$. In order to estimate the contribution from $S$ we need the following lemma.

LEMMA 8. For $|\alpha| \leq 1 / 2$ and any positive integers $m$ and $k$ such that $k \geq 2$ we have

$$
\sum_{j=m}^{m+k-1} 2 \sin ^{2} \pi \alpha j \geq \frac{k}{2} \min \left\{1,(\alpha k)^{2}\right\}
$$

PROOF. Clearly we can assume without loss of generality that

$$
0<\alpha \leq 1 / 2
$$

We have

$$
\sum_{j=m}^{m+k-1} 2 \sin ^{2} \pi \alpha j=\sum_{j=m}^{m+k-1}(1-\cos 2 \pi \alpha j) \geq k-\left|\sum_{j=m}^{m+k-1} \cos 2 \pi \alpha j\right| \geq k-\frac{|\sin \pi \alpha k|}{\sin \pi \alpha} .
$$

If $1 / k \leq \alpha \leq 1 / 2$, then

$$
\sin \pi \alpha \geq \frac{2}{\pi} \pi \alpha=2 \alpha \geq \frac{2}{k},
$$

and so the right-hand side of (5.2) is

$$
\geq \frac{k}{2}=\frac{k}{2} \min \left(1,(\alpha k)^{2}\right) .
$$

If $0<\alpha<1 / k$, then $0<\alpha \leq \alpha k / 2<1 / 2$ and $\operatorname{since} \sin \pi x / \pi x$ is strictly decreasing on $(0,1 / 2)$ we have

$$
0 \leq \sin (\pi \alpha k / 2) \leq(k / 2) \sin \pi \alpha, \quad \sin (\pi \alpha k / 4) \geq \alpha k / 2
$$

thus in this case the right-hand side of (5.2) is

$$
\begin{aligned}
& \geq k-\frac{2 \sin (\pi \alpha k / 2) \cos (\pi \alpha k / 2)}{\sin \pi \alpha} \\
& \geq k\left(1-\cos \pi \alpha \frac{k}{2}\right)=2 k \sin ^{2} \pi \alpha \frac{k}{4} \\
& \geq 2 k\left(\frac{\alpha k}{2}\right)^{2}=\frac{k}{2}(\alpha k)^{2}=\frac{k}{2} \min \left\{1,(\alpha k)^{2}\right\} .
\end{aligned}
$$

Thus in both cases the stated inequality holds. 
We can now estimate the contribution from $S$.

LEMMA 9. Suppose $n \geq e^{8}, 1 \leq m \leq n / 2$ and $\alpha_{0} \leq \sigma$; then for $S$ as in (5.1) we have

$$
\left|\int_{S} e^{-2 \pi i \alpha n} \varphi(\alpha) d \alpha\right| \leq \exp \left(-(32 e)^{-1} e^{-\sigma m} \alpha_{0}^{2} \sigma^{-3}\right) .
$$

Proof. Consider $\alpha$ in $S$. We note that by Lemma 2(i) and Lemma 2(ii) (with $r=[n / 2]$ ) and our choice of $n$ we have $\sigma^{-1}>2$ and $\sigma n>2$. We consider the integer $k$ given by $k=\left[\sigma^{-1}\right]$, noting that $k \leq n / 2$ and hence $m+k \leq n$. By (i) of the corollary to Lemma 3 we have

$$
|\varphi(\alpha)| \leq \exp \left(-2 \sum_{j=m}^{m+k-1} p_{1 j} p_{2 j} \sin ^{2} \pi \alpha j\right)
$$

and since $\sigma>0$ it follows from (2.5) that

$$
|\varphi(\alpha)| \leq \exp \left(-\frac{1}{2} e^{-\sigma(m+k)} \sum_{j=m}^{m+k-1} \sin ^{2} \pi \alpha j\right) .
$$

Now by Lemma 8 , since $\alpha$ is in $S$ and $k \geq 2$ we have

$$
\sum_{j=m}^{m+k-1} \sin ^{2} \pi \alpha j \geq \frac{k}{2} \min \left\{1,(\alpha k)^{2}\right\} \geq \frac{k}{2} \min \left\{1,\left(\alpha_{0} k\right)^{2}\right\} \geq \frac{1}{2} \alpha_{0}^{2} k^{3},
$$

since $\alpha_{0} k \leq \alpha_{0} \sigma^{-1} \leq 1$. Also by our choice of $k$, we have $e^{-\sigma k} \geq e^{-1}$ and $k \geq \sigma^{-1} / 2$. Thus we obtain

$$
|\varphi(\alpha)| \leq \exp \left(-(32 e)^{-1} e^{-\sigma m} \alpha_{0}^{2} \sigma^{-3}\right) .
$$

Since $\left|e^{-2 \pi i n \alpha}\right|=1$ and $S$ has total length less than 1, integration over $S$ now gives the result stated.

\section{Completion of the proof of Theorem 1}

For positive $X$ and $Y$ we shall write $X \ll Y$ to mean that $X \leq C Y$ if $n \geq n_{0}$, where $C$ and $n_{0}$ are effective positive constants, independent of $m, n$ and $\alpha_{0}$, and all constants mentioned will satisfy this requirement.

Suppose now that $n \geq e^{8}, 1 \leq m \leq n / 2$ and

$$
(2 \pi B)^{-1} \leq \alpha_{0} \leq \min \left(\sigma(3 \pi)^{-1},\left(\rho_{3}\right)^{-1 / 3}\right),
$$


and hence that the condition $\alpha_{0} \leq \sigma$ in Lemma 9 is also satisfied. Then Lemmas 4 and 9 show that for $J$ as in (2.10) we have

$$
J=\left(2 \pi B^{2}\right)^{-1 / 2}(1+E),
$$

where $E=E(m, n)$ satisfies

$$
E \ll e^{-2 \pi^{2} \alpha_{0}^{2} B^{2}}+\alpha_{0}^{3} \rho_{3}+B \exp \left\{-(32 e)^{-1} e^{-\sigma m} \alpha_{0}^{2} \sigma^{-3}\right\} .
$$

First we need a lower bound for $e^{-\sigma m} \alpha_{0}^{2} \sigma^{-3}$. Let $K$ be the constant in Lemma 7(i). If $K \sigma^{-1}<m \leq n / 2$, then by Lemma 7(i)

$$
e^{-\sigma m} \alpha_{0}^{2} \sigma^{-3}=\frac{m^{2} e^{-\sigma m}}{\sigma} \cdot \frac{1}{(\sigma m)^{2}} \cdot \alpha_{0}^{2} \gg \frac{B^{2} \cdot \alpha_{0}^{2}}{(\log n)^{2}} .
$$

On the other hand, if $1 \leq m \leq K \sigma^{-1}$, then by Lemma 6(i)

$$
e^{-\sigma m} \alpha_{0}^{2} \sigma^{-3} \gg e^{-K} \alpha_{0}^{2} B^{2} \gg \frac{B^{2} \cdot \alpha_{0}^{2}}{(\log n)^{2}} .
$$

Since $B \geq 1$ by (6.1), the error $E$ in (6.2) satisfies

$$
E \ll \alpha_{0}^{3} \rho_{3}+B \exp \left\{-\left(\frac{\alpha_{0} B}{C \log n}\right)^{2}\right\},
$$

where $C$ is an effective positive constant.

We seek to choose $\alpha_{0}$ so as to equalise the two expressions on the right-hand side of (6.3), so that

$$
\exp \left\{-\left(\frac{\alpha_{0} B}{C \log n}\right)^{2}\right\}=\alpha_{0}^{3} \frac{\rho_{3}}{B}=\left(\frac{\alpha_{0} B}{C \log n}\right)^{3} \frac{1}{\gamma},
$$

where

$$
\gamma=\frac{B^{4}}{(C \log n)^{3} \rho_{3}}
$$

By the Corollary to Lemma 7

$$
n(\log n)^{-3} \ll \gamma \ll n(\log n)^{-3},
$$

and hence $\gamma \geq e$ provided $n \geq n_{1}$, for suitable constant $n_{1}$. From now on we assume that $n \geq n_{1}$ and we take

$$
\alpha_{0}=\frac{C x_{0}}{B} \log n,
$$


where $x=x_{0}$ satisfies $e^{-x^{2}}=x^{3} / \gamma$, that is $x^{3} e^{x^{2}}=\gamma$. It is easily seen that $x_{0}$ is unique and

$$
(\log \gamma)^{1 / 2} / 3<x_{0}<(\log \gamma)^{1 / 2}
$$

and hence

$$
(\log n)^{1 / 2} \ll x_{0} \ll(\log n)^{1 / 2} .
$$

We must check (6.1). Clearly (6.4) and (6.5) imply that $\alpha_{0} \geq(2 \pi B)^{-1}$ provided $n \geq n_{2}$, for suitable $n_{2}$. We now look at upper bounds for $\alpha_{0}$.

If $1 \leq m \leq K \sigma^{-1}$ then by Lemmas 6.4 and 6.5, and Lemmas 7(ii) and 6(i) we have

$$
\frac{\alpha_{0}}{\sigma} \ll \frac{(\log n)^{3 / 2}}{\sigma B} \ll \frac{(\log n)^{3 / 2}}{\sigma^{-1 / 2}} \ll \frac{(\log n)^{3 / 2}}{n^{1 / 4}}
$$

and

$$
\alpha_{0}^{3} \rho_{3} \ll(\log n)^{9 / 2} \rho_{3} B^{-3} \ll \frac{(\log n)^{9 / 2}}{n^{1 / 4}} .
$$

On the other hand, if $K \sigma^{-1}<m \leq n / 2$ then it follows from Lemma 7(i) that

$$
B^{2} \gg m n, \quad \rho_{3} \ll m B^{2} \ll m^{2} n,
$$

and in this case we obtain

$$
\begin{aligned}
& \alpha_{0} / \sigma \ll(\log n)^{3 / 2} m / B \ll(\log n)^{3 / 2}(m / n)^{1 / 2}, \\
& \alpha_{0}^{3} \rho_{3} \ll(\log n)^{9 / 2} \rho_{3} B^{-3} \ll(\log n)^{9 / 2}(m / n)^{1 / 2} .
\end{aligned}
$$

It follows from the above estimates that there is a positive constant $K_{0}$ such that (6.1) holds for $n$ sufficiently great provided that $1 \leq m \leq K_{0} n(\log n)^{-9}$. By (6.3) and our choice of $x_{0}$, the error $E$ in (6.2) then satisfies $E \ll \alpha_{0}^{3} \rho_{3}$ and combining our upper bounds for $\alpha_{0}^{3} \rho_{3}$ we obtain

$$
E \ll(\log n)^{9 / 2} \max \left\{\frac{1}{n^{1 / 4}},\left(\frac{m}{n}\right)^{1 / 2}\right\},
$$

that is, $E$ satisfies (2.8). By the remarks at the end of Section 2 this completes the proof of Theorem 1 .

\section{Further detailed estimates}

In order to obtain explicit asymptotic estimates in terms of $m$ and $n$ for $q_{m}(n)$, we must look in more detail at the main term of the estimate of $q_{m}(n)$ in Theorem 1. For 
this purpose we clearly need to estimate the numerator

$$
\begin{aligned}
e^{\sigma n} \prod_{j=m}^{n}\left(1+e^{-j \sigma}\right) & =\exp \left\{\sigma n+\sum_{j=m}^{n} \log \left(1+e^{-j \sigma}\right)\right\} \\
& =\exp \left\{\sum_{j=m}^{n}\left(\frac{\sigma j}{1+e^{\sigma j}}+\log \left(1+e^{-j \sigma}\right)\right)\right\}
\end{aligned}
$$

We also need an asymptotic estimate for $B^{2}$ when $m$ is relatively small (significantly less than $\sqrt{n}$ ), and we need more precise information about $\sigma$, which we obtain by more careful study of the sum (2.7) which determines $\sigma$. In this section we shall obtain the necessary estimates, which will be based on the following lemma.

LEMMA 10. Suppose $1 \leq r \leq s$. Then we have the following estimates, with all implied constants effective and independent of $r, s, m$ and $n$.

Let

$$
f(x)=\frac{\sigma x}{1+e^{\sigma x}}+\log \left(1+e^{-\sigma x}\right) .
$$

Then

(i)

$$
\begin{aligned}
& \left.\sum_{j=r}^{s} f(j)=2 \int_{r-1}^{s} \frac{\sigma x}{1+e^{\sigma x}} d x+x \log \left(1+e^{-\sigma x}\right)\right]_{r-1}^{s} \\
& \quad+(f(s)-f(r-1)) / 2+O(\sigma)+O\left(\sigma^{2} s\right)
\end{aligned}
$$

(ii)

$$
\begin{aligned}
& \text { (ii) } \left.\sum_{j=r}^{s} \frac{\sigma j}{1+e^{\sigma j}}=\int_{r-1}^{s} \frac{\sigma x}{1+e^{\sigma x}} d x+\frac{\frac{1}{2} \sigma x}{1+e^{\sigma x}}\right]_{r-1}^{s}+O(\sigma)+O\left(\sigma^{2} s\right) \\
& \text { (iii) } \left.\sum_{j=r}^{s} \frac{\sigma^{2} j^{2} e^{\sigma j}}{\left(1+e^{\sigma j}\right)^{2}}=\int_{r-1}^{s} \frac{\sigma^{2} x^{2} e^{\sigma x}}{\left(1+e^{\sigma x}\right)^{2}} d x+\frac{\frac{1}{2} \sigma^{2} x^{2} e^{\sigma x}}{\left(1+e^{\sigma x}\right)^{2}}\right]_{r-1}^{s}+O(\sigma)+O\left(\sigma^{2} s\right) .
\end{aligned}
$$

PROOF. (i) For $x>0$, we have

$$
f^{\prime}(x)=-\sigma^{2} x e^{\sigma x}\left(1+e^{\sigma x}\right)^{-2}<0,
$$

so that $f$ is strictly decreasing on $(0, \infty)$. Since $\sigma>0$, we have $e^{\sigma x}>\sigma x \geq 0$ for $x \geq 0$. Thus it is clear that

$$
f^{\prime}(x)=O(\sigma) \quad \text { for } x \geq 0 \text {, }
$$

and it is also easily checked that

$$
f^{\prime \prime}(x)=O\left(\sigma^{2}\right) \quad \text { for } x \geq 0 .
$$


We use the Euler-Maclaurin summation formula in the form

$\left.\left.\sum_{j=r}^{s} f(j)=\int_{r-1}^{s} f(x) d x+\frac{1}{2} f(x)\right]_{r-1}^{s}+\frac{B_{2}}{2} f^{\prime}(x)\right]_{r-1}^{s}-\frac{1}{2} \int_{r-1}^{s} B_{2}(x-[x]) f^{\prime \prime}(x) d x$, where $B_{2}(t)=t^{2}-t+\frac{1}{6}$ and $B_{2}=B_{2}(0)$, so that $B_{2}(x-[x])=O(1)$. The required estimate (i) then follows by integrating $\log \left(1+e^{-\sigma x}\right)$ by parts and using (7.3) and (7.4). Estimates corresponding to (7.3) and (7.4) hold for the functions involved in the two sums in (ii) and (iii) and the required conclusions follow in a similar way (except that no integration by parts is required).

First we use Lemma 10 to give the estimates which are needed when $m$ is relatively large.

LEMMA 11. As $n \rightarrow \infty$, the following estimates hold uniformly with respect to $m$ such that

$$
6 n^{1 / 2} \leq m \leq K_{0} n(\log n)^{-9},
$$

with $K_{0}$ as in Theorem 1 and all implied constants effective and independent of $m$ and $n$.

(i) We have

$$
\begin{aligned}
e^{\sigma n} \prod_{j=m}^{n}\left(1+e^{-\sigma j}\right)=\exp \left\{\frac{2}{\sigma} \int_{\sigma m}^{\infty} \frac{y}{1+e^{y}} d y-m \log \left(1+e^{-\sigma m}\right)\right. \\
\left.+O\left(\sigma(\log n)^{3}\right)+O\left(\sigma m e^{-\sigma m}\right)\right\} .
\end{aligned}
$$

(ii) We have

$$
\sigma n=\frac{1}{\sigma} \int_{\sigma m}^{\infty} \frac{y}{1+e^{y}} d y+O\left(\sigma(\log n)^{3}+O\left(\sigma m e^{-\sigma m}\right)\right) .
$$

Proof. We assume that (7.5) holds and note that, by Lemma 2(ii),

$$
\sigma m>\log \left(\frac{m}{2 \sqrt{ } n}\right) \geq 1
$$

Also for $f(x)$ as in (7.2) and $x \geq m$ we have

$$
f(x)=O\left(\sigma x e^{-\sigma x}\right)=O\left(\sigma m e^{-\sigma m}\right) .
$$

By (7.1) and (7.2),

$$
e^{\sigma n} \prod_{j=m}^{n}\left(1+e^{-\sigma j}\right)=\exp \left\{\sum_{j=m}^{n} f(j)\right\}=\exp S,
$$


say. We split $S$ into two sums,

$$
S=\sum_{j=m}^{s} f(j)+\sum_{j=s+1}^{n} f(j)=S_{1}+S_{2}
$$

say, where $s=\left[2 m(\log n)^{2}\right]$, which is less than $n$ for large $n$ by (7.5). For sufficiently large $n$ we have $\sigma s>\sigma m(\log n)^{2}>(\log n)^{2}$, and hence

$$
S_{2}=O\left(n(\log n)^{2} e^{-(\log n)^{2}}\right)
$$

We now apply Lemma 10 (i) to $S_{1}$, with $r-1=m$, and use the change of variable $\sigma x=y$ on the relevant integral, obtaining

$$
S_{1}=\frac{2}{\sigma} \int_{\sigma m}^{\sigma s} \frac{y}{1+e^{y}} d y-m \log \left(1+e^{-\sigma m}\right)+O\left(s e^{-\sigma s}+f(m)+\sigma+\sigma^{2} s\right) .
$$

Also we note that

$$
\frac{1}{\sigma} \int_{\sigma s}^{\infty} \frac{y}{1+e^{y}} d y=O\left(s e^{-\sigma s}\right)=O\left(n(\log n)^{2} e^{-(\log n)^{2}}\right) .
$$

Using (7.5), (7.6) and (7.7) and the estimate $\sigma m<\log n$ from Lemma 7(i), we see that as $n \rightarrow \infty$

$$
S=S_{1}+S_{2}=\frac{2}{\sigma} \int_{\sigma m}^{\infty} \frac{y}{1+e^{y}} d y-m \log \left(1+e^{-\sigma m}\right)+E,
$$

where

$$
E=O\left(\sigma(\log n)^{3}\right)+O\left(\sigma m e^{-\sigma m}\right) .
$$

This completes the proof of (i).

The proof of (ii) is similar, starting from

$$
n \sigma=\sum_{j=m}^{n} \frac{\sigma j}{1+e^{\sigma j}},
$$

splitting the sum into two in exactly the same way as before, and using Lemma 7(ii) on the first sum.

COROLLARY. Under the conditions of Lemma 11, we have

$$
e^{\sigma n} \prod_{n=m}^{n}\left(1+e^{-\sigma j}\right)=\exp \left\{2 \sigma n-m \log \left(1+e^{-\sigma m}\right)+O\left(\sigma(\log n)^{3}\right)+O\left(\sigma m e^{-\sigma m}\right)\right\}
$$


PROOF. Use (ii) to replace the integral in (i).

Now we give the corresponding estimates needed when $m$ is relatively small.

LEMMA 12. Let $f(x)$ be as in (7.2). As $n \rightarrow \infty$, the following estimates hold uniformly with respect to $m$ such that

$$
1 \leq m \leq n^{1 / 2},
$$

with all implied constants effective and independent of $m$ and $n$. We have

$$
\begin{aligned}
\sum_{j=1}^{n} f(j) & =\frac{\pi^{2}}{6 \sigma}-\frac{1}{2} \log 2+O\left(\sigma(\log n)^{3}\right) ; \\
\sum_{j=1}^{n} \frac{\sigma j}{1+e^{\sigma j}} & =\frac{\pi^{2}}{12 \sigma}+O\left(\sigma(\log n)^{3}\right) ; \\
\sum_{j=1}^{n} \frac{(\sigma j)^{2} e^{\sigma j}}{\left(1+e^{\sigma j}\right)^{2}} & =\frac{\pi^{2}}{6 \sigma}+O\left(\sigma(\log n)^{3}\right) .
\end{aligned}
$$

ProOF. We assume now that (7.8) holds, and note that, by Lemma 2(i), $m \sigma \leq 2$, so that for $K$ as in Lemma 7 we have

$$
m \leq K \sigma^{-1} .
$$

By Lemma 7(ii) it follows that for $n$ sufficiently great and $C$ as in Lemma 7

$$
\sigma \geq C^{1 / 2} n^{-1 / 2}=D n^{-1 / 2},
$$

say, where $D=C^{1 / 2}>0$.

Again we split our sum into two parts,

$$
S=\sum_{j=1}^{s} f(j)+\sum_{j=s+1}^{n} f(j)=S_{1}+S_{2},
$$

say, where $s=\left[2 n^{1 / 2}(\log n)^{2}\right]$, so that $\sigma s>D(\log n)^{2}$, and hence

$$
S_{2}=O\left(n D(\log n)^{2} e^{-D(\log n)^{2}}\right)=O\left(\sigma(\log n)^{3}\right),
$$

using $\sigma \geq D n^{-1 / 2}$. We note that now

$$
\frac{1}{\sigma} \int_{\sigma s}^{\infty} \frac{y}{1+e^{y}} d y=O\left(s e^{-\sigma s}\right)=O\left(n^{1 / 2}(\log n)^{2} e^{-D(\log n)^{2}}\right)=O\left(\sigma(\log n)^{3}\right) .
$$


This time we apply Lemma 10(i) to $S_{1}$ with $r-1=0$. Arguing as in the proof of Lemma 11(i) and noting that $f(0)=\log 2$ we obtain

$$
S_{1}=\frac{2}{\sigma} \int_{0}^{\infty} \frac{y}{1+e^{y}} d y-\frac{1}{2} \log 2+o\left(s e^{-\sigma s}+f(s)+\sigma+\sigma^{2} s\right),
$$

where by using the above estimates and the fact $\sigma<2 n^{-1 / 2}$ by Lemma 2(i), we see that the error term is $O\left(\sigma(\log n)^{3}\right)$. Combining the above results, we obtain

$$
S=\frac{2}{\sigma} \int_{0}^{\infty} \frac{y}{1+e^{y}} d y-\frac{1}{2} \log 2+O\left(\sigma(\log n)^{3}\right) .
$$

By writing the integrand in the form $y e^{-y} \sum_{j=0}^{\infty}(-1)^{j} e^{-j y}$ it is easily shown that

$$
\int_{0}^{\infty} \frac{y}{1+e^{y}} d y=\frac{1}{2} \zeta(2)=\frac{\pi^{2}}{12}
$$

and this completes the proof of (i).

The proofs of (ii) and (iii) are similar, using the same splitting of the sums with respect to the same $s$ together with parts (ii) and (iii) of Lemma 10. To evaluate the main term in (iii), we also need to use integration by parts and (7.9) to show that

$$
\left.\int_{0}^{\infty} \frac{y^{2} e^{y}}{\left(1+e^{y}\right)^{2}} d y=-\frac{y^{2}}{1+e^{y}}\right]_{0}^{\infty}+2 \int_{0}^{\infty} \frac{y}{1+e^{y}} d y=\frac{\pi^{2}}{6} .
$$

\section{Explicit estimate of $q_{m}(n)$ for large $m$}

We now use the corollary to Theorem 1 and the relevant estimates from Section 7 to obtain an explicit asymptotic estimate for $q_{m}(n)$ which is valid for $m$ relatively large compared with $n^{1 / 2}$. We therefore assume throughout this section that

$$
n^{1 / 2} \psi(n) \leq m \leq K_{0} n(\log n)^{-10},
$$

where $K_{0}$ is as in Theorem 1 and $(\psi(n))$ is a strictly increasing sequence such that as $n \rightarrow \infty$

$$
\psi(n) \rightarrow \infty, \quad \psi(n)=o\left(n^{1 / 2}(\log n)^{-10}\right) .
$$

We note that then, by Lemma 2 ,

$$
\frac{1}{m} \log (\psi(n) / 2)<\sigma<2 n^{-1 / 2} .
$$


It follows that under the above conditions as $n \rightarrow \infty$ we have

$$
\sigma(\log n)^{3} \rightarrow 0, \quad \sigma m \rightarrow \infty .
$$

Hence, as $n \rightarrow \infty$,

$$
\sigma m e^{-\sigma m} \rightarrow 0, \quad e^{-\sigma m} \rightarrow 0,
$$

and, by Lemma 6(ii) $B^{2} \sim m n$.

By the corollary to Theorem 1, we see that under the above conditions as $n \rightarrow \infty$

$$
q_{m}(n) \sim \frac{1}{\sqrt{ }(2 \pi m n)} e^{\sigma n} \prod_{j=m}^{n}\left(1+e^{-\sigma j}\right),
$$

and it then follows from Lemma 11(i) that

$$
q_{m}(n) \sim \frac{1}{\sqrt{ }(2 \pi m n)} \exp \left\{\frac{2}{\sigma} \int_{\sigma m}^{\infty} \frac{y}{1+e^{y}} d y-m \log \left(1+e^{-\sigma m}\right)\right\} .
$$

For $X>0$, we define

$$
\begin{aligned}
& F(X)=\frac{1}{X^{2}} \int_{X}^{\infty} \frac{y}{1+e^{y}} d y \\
& G(X)=2 X F(X)-\log \left(1+e^{-X}\right) .
\end{aligned}
$$

Then as $n \rightarrow \infty$ we have

$$
q_{m}(n) \sim \frac{1}{\sqrt{ }(2 \pi m n)} \exp \left\{2 m^{2} \sigma F(\sigma m)-m \log \left(1+e^{-\sigma m}\right)\right\},
$$

that is,

$$
q_{m}(n) \sim \frac{1}{\sqrt{ }(2 \pi m n)} \exp \{m G(\sigma m)\} .
$$

In order to estimate the right-hand side, we use Lemma 11 to obtain an estimate of $\sigma m$ as follows. By Lemma 11(ii) under the above conditions we have

$$
\sigma n=\frac{1}{\sigma} \int_{\sigma m}^{\infty} \frac{y}{1+e^{y}} d y+O\left(\sigma(\log n)^{3}\right)+O\left(\sigma m e^{-\sigma m}\right)
$$

and hence, dividing by $\sigma \mathrm{m}^{2}$ and using (8.3),

$$
n / m^{2}=F(\sigma m)+O\left((\log n)^{3} / m^{2}\right)+O\left(e^{-\sigma m} / m\right) .
$$

It is easily checked that $F^{\prime}(X)<0$ for $X>0$, so that $F$ is strictly decreasing and hence invertible. Equation (8.6) shows that $F^{-1}\left(n / m^{2}\right)$ approximates $\sigma m$, and we shall show that this approximation is good enough to be used in (8.5). We can now formulate our result as follows. 
THEOREM 2. Let $F$ be as in (8.3), and let $(\psi(n))$ be an increasing sequence such that $\psi(n) \rightarrow \infty$ as $n \rightarrow \infty$ and $\psi(n)=o\left(n^{1 / 2}(\log n)^{-10}\right)$. Then as $n \rightarrow \infty$ we have

$$
q_{m}(n) \sim \frac{1}{\sqrt{ }(2 \pi m n)} \exp \left\{\frac{2 n}{m} F^{-1}\left(\frac{n}{m^{2}}\right)-m \log \left(1+e^{-F^{-1}\left(n / m^{2}\right)}\right)\right\},
$$

uniformly with respect to $m$ such that

$$
\psi(n) \sqrt{n} \leq m \leq K_{0} n(\log n)^{-10},
$$

where $K_{0}$ is the constant in Theorem 1, while all other implied constants are effective and independent of $m$ and $n$ (but depend on $\psi$ ).

Before proving the theorem it is convenient to gather together some properties of the functions $F$ and $G$ in the following lemma.

LEMMA 13. For $F$ and $G$ as in (8.3) and (8.4) we have the following results, in which the implied constants are independent of the parameters involved $(a, b, X)$.

(i) Let $a$ and $b$ be positive real numbers such that

$$
|b-a|<a / 2, \quad F^{-1}(a) \geq 1, \quad F^{-1}(b) \geq 1 .
$$

Then

$$
\left|F^{-1}(b)-F^{-1}(a)\right| \ll|b-a| / a .
$$

(ii) Let $a$ and $b$ be positive real numbers such that $|b-a|<a / 2$. Then

$$
|G(b)-G(a)| \ll a|F(b)-F(a)| .
$$

(iii) For $X \geq 1$

$$
\begin{aligned}
& F(X)=\left(\frac{1}{X}+\frac{1}{X^{2}}\right) e^{-X}+O\left(e^{-2 X}\right) \\
& G(X)=\left(1+\frac{2}{X}\right) e^{-X}+O\left(e^{-2 X}\right)=X F(X) \frac{X+2}{X+1}+O\left(X e^{-2 X}\right) .
\end{aligned}
$$

(iv) As $Y \rightarrow 0, F^{-1}(Y) \sim \log (1 / Y)$.

PROOF. (i) By the mean value theorem

$$
F^{-1}(b)-F^{-1}(a)=(b-a) / F^{\prime}\left(F^{-1}(c)\right)
$$

for some $c$ between $a$ and $b$. It is easily checked that $F^{\prime \prime}(X)>0$ for $X>0$ (so that $F^{\prime}$ is strictly monotonic) and that for $X \geq 1 F(X) \ll\left|F^{\prime}(X)\right| \ll F(X)$. Therefore $\left|F^{\prime}\left(F^{-1}(c)\right)\right| \gg F\left(F^{-1}(c)\right)=c \gg a$, and the required inequality follows.

(ii) By Cauchy's mean value theorem

$$
G(b)-G(a)=(F(b)-F(a)) G^{\prime}(c) / F^{\prime}(c)
$$


for some $c$ between $a$ and $b$. It is easily checked that for $X>0$ we have $G^{\prime}(X)=$ $X F^{\prime}(X)$. Hence $G^{\prime}(c) / F^{\prime}(c)=c \ll a$.

(iii) The first result follows from (8.3) by using the estimate

$$
\frac{1}{1+e^{y}}=e^{-y}\left(1+e^{-y}\right)^{-1}=e^{-y}+O\left(e^{-2 y}\right)
$$

and integrating. The second then follows from (8.4) by using the estimate

$$
\log \left(1+e^{-X}\right)=e^{-X}+O\left(e^{-2 X}\right) .
$$

(iv) It is easily seen that $F^{-1}(Y) \rightarrow \infty$ as $Y \rightarrow 0$. From (iii) we have

$$
X e^{X}=\frac{1}{Y}\left(1+\frac{1}{X}+O\left(X e^{-X}\right)\right),
$$

where $X=F^{-1}(Y)$, and we obtain (iv) by considering logarithms.

We can now prove Theorem 2. By Lemma 13(i) with $a=n / m^{2}$ and $b=F(\sigma m)$, we obtain from (8.6) the estimate

$$
\sigma m=F^{-1}\left(n / m^{2}\right)+O\left((\log n)^{3} / n\right)+O\left(m e^{-\sigma m} / n\right),
$$

in which the error terms tend to zero as $n \rightarrow \infty$. By (ii) with $a=\sigma m$ and $b=F^{-1}\left(n / m^{2}\right)$, we then obtain from (8.6) the estimate

$$
m\left\{G(\sigma m)-G\left(F^{-1}\left(n / m^{2}\right)\right)\right\}=O\left(\sigma(\log n)^{3}\right)+O\left(\sigma m e^{-\sigma m}\right),
$$

which tends to zero as $n \rightarrow \infty$ under our assumptions, by (8.1) and (8.2). Hence as $n \rightarrow \infty$

$$
\exp (m G(\sigma m)) \sim \exp \left(m G\left(F^{-1}\left(n / m^{2}\right)\right),\right.
$$

and the theorem now follows from (8.4) and (8.5).

If $Y=n / m^{2}$ and $X=F^{-1}(Y)$ then it follows from our assumptions and Lemma 13(iii), (iv) that as $n \rightarrow \infty, e^{-X} \sim X Y$, and

$$
m e^{-2 X} \sim m(X Y)^{2} \ll(\log n)^{2} n^{2} / m^{3} .
$$

If $m \geq(\log n) n^{2 / 3}$ it follows that $m e^{-2 X}=o(1)$ and so we obtain the following corollary from Lemma 13(iii).

COROLlaRY 1. As $n \rightarrow \infty$ we have

$$
q_{m}(n) \sim \frac{1}{\sqrt{ }(2 \pi m n)} \exp m\left\{\left(1+\frac{2}{F^{-1}\left(n / m^{2}\right)}\right) e^{-F^{-1}\left(n / m^{2}\right)}\right\}
$$


uniformly with respect to $m$ such that

$$
n^{2 / 3} \log n \leq m \leq K_{0} n(\log n)^{-10},
$$

where $K_{0}$ is the constant in Theorem 1, while all other implied constants are effective and independent of $m$ and $n$.

For fixed $\alpha>1 / 3$, we can obtain a precise estimate for $G\left(F^{-1}\left(n / m^{2}\right)\right)$ by restricting $m$ to a range in which $\alpha \log n$ is a reasonable approximation to $F^{-1}\left(n / m^{2}\right)$. We note that by Lemma 13(iii)

$$
F(\alpha \log n)=F_{1}+O\left(n^{-2 \alpha}\right),
$$

where

$$
F_{1}=F_{1}(\alpha, n)=\left\{\frac{1}{\alpha \log n}+\frac{1}{(\alpha \log n)^{2}}\right\} n^{-\alpha} .
$$

If $m=\left(n / F_{1}\right)^{1 / 2}+E$, with suitable $E$, then Lemma 13(ii) will yield

$$
\begin{aligned}
m\left(G\left(F^{-1}\left(n / m^{2}\right)\right)-G(\alpha \log n)\right. & \ll m \alpha \log n\left|\frac{n}{m^{2}}-F_{1}+O\left(n^{-2 \alpha}\right)\right| \\
& \ll \frac{\alpha \log n}{m} F_{1} E(2 m+E)+m \alpha n^{-2 \alpha} \log n \\
& \ll E n^{-\alpha}+m \alpha \log n n^{-2 \alpha} .
\end{aligned}
$$

If $E=o\left(n^{\alpha}\right)$ then $E n^{-\alpha} \rightarrow 0$ as $n \rightarrow \infty$, and for $m$ as above we have

$$
m \ll(\alpha \log n)^{1 / 2} n^{(1+\alpha) / 2} .
$$

The condition $\alpha>1 / 3$ then ensures that as $n \rightarrow \infty, m \alpha \log n n^{-2 \alpha} \rightarrow 0$. These considerations lead to the following further corollary.

COROLlaRY 2. Suppose $1 / 3<\alpha<1$. Then as $n \rightarrow \infty$

$$
q_{m}(n) \sim \frac{1}{\sqrt{ }(2 \pi m n)} \exp \left\{\frac{m}{n^{\alpha}}\left(1+\frac{2}{\alpha \log n}\right)\right\}
$$

uniformly with respect to $m$ such that

$$
m=\frac{\alpha \log n}{(1+2 \log n)^{1 / 2}} n^{(1+\alpha) / 2}+o\left(n^{\alpha}\right) .
$$

(More precisely, let $\left(\theta_{n}\right)$ be a decreasing sequence of positive numbers such that $\theta_{n} \rightarrow 0$ as $n \rightarrow \infty$ and let $F_{1}$ be as in (8.8). Then (8.9) holds uniformly with respect to $m$ such that

$$
\left|m-\left(n / F_{1}\right)^{1 / 2}\right| \leq \theta_{n} n^{\alpha},
$$

with all implied constants independent of $m, n$ and $\alpha$ (but dependent on the sequence $\left.\left(\theta_{n}\right)\right)$.) 
PROOF. It is easily checked that the conditions on $\alpha$ and $m$ ensure that Theorem 2 applies and that the above argument is valid. Thus

$$
m\left(G\left(F^{-1}\left(n / m^{2}\right)\right)-G(\alpha \log n)\right) \rightarrow 0 \quad \text { as } n \rightarrow \infty .
$$

Also by Lemma 13(iii)

$$
m G(\alpha \log n)=m\left(1+2(\alpha \log n)^{-1}\right) n^{-\alpha}+O\left(m n^{-2 \alpha}\right),
$$

and our conditions ensure that as $n \rightarrow \infty, m n^{-2 \alpha} \rightarrow 0$. Thus as $n \rightarrow \infty$

$$
\exp \left\{m G\left(F^{-1}\left(n / m^{2}\right)\right)\right\} \sim \exp \left\{m\left(1+2(\alpha \log n)^{-1}\right) n^{-\alpha}\right\},
$$

and the corollary follows from Theorem 2 .

We note that under the conditions of the corollary, (8.9) implies that as $n \rightarrow \infty$

$$
q_{m}(n) \sim \frac{1}{\sqrt{ }(2 \pi) n^{(\alpha+3) / 4}} \exp \left\{n^{(1-\alpha) / 2} \frac{2+\alpha \log n}{(1+\alpha \log n)^{1 / 2}}\right\} .
$$

Instead of restricting the range of $m$ and using the rough estimate of Lemma 13(ii) with $b=F^{-1}\left(n / m^{2}\right)$ we might estimate $F^{-1}\left(n / m^{2}\right)$ by more precise methods, giving, say

$$
F^{-1}\left(n / m^{2}\right)=\Phi\left(n / m^{2}\right)+E .
$$

We can see how small $E$ needs to be as follows. If, further, $m \geq(\log n)^{2} n^{2 / 3}$, then, by using the second expression in Lemma 13(iii) and Corollary 1 we see that

$$
q_{m}(n) \sim \frac{1}{\sqrt{ }(2 \pi m n)} \exp \left\{\frac{n}{m} \frac{X(X+2)}{X+1}\right\},
$$

where $X=F^{-1}\left(n / m^{2}\right)$. In order to use (8.10) to obtain an asymptotic estimate by replacing $X$ by $\Phi\left(n / m^{2}\right)$ we will clearly need $E=o(m / n)$ as $n \rightarrow \infty$.

By classical methods similar to those used by Dixmier and Nicolas [2] for $H(x)$, it is possible to obtain an expansion of $X$ in the form

$$
X=F^{-1}(Y)=\frac{1}{v}+L+\sum_{j=1}^{k-1} P_{j}(L) v^{j}+O\left(v^{k}|L|^{k}\right),
$$

where

$$
Y=n / m^{2}, \quad v=\left(\log \left(m^{2} / n\right)\right)^{-1} \geq(\log n)^{-1}, \quad L=\log v .
$$


Professor Nicolas has kindly sent this information together with his calculations of $P_{1}(L), P_{2}(L), \ldots, P_{9}(L)$. For example, for $k=3$ the expansion is

$$
X=\frac{1}{v}+L+(1-L) v+\left(\frac{1}{2} L^{2}-\frac{3}{2}\right) v^{2}+O\left(v^{3}|L|^{3}\right) .
$$

In order to ensure that under our conditions $v^{k}|L|^{k}=o(m / n)$ we would need something like $m \gg v^{k-1} n$, that is, roughly $m \gg n(\log n)^{-(k-1)}$. Thus taking $k=11$ would yield a suitable estimate $X$ when $m$ is at the extreme right-hand end of the range in Corollary 1 . We omit the details since the required value of $k$ can be reduced by extending the range for $m$ further to the right. Separate detailed consideration of the right-hand end of the range will be pursued further elsewhere.

\section{Explicit estimate of $q_{m}(n)$ for small $m$}

We now consider the case when $m$ is relatively small compared with $n^{1 / 2}$. By Theorem 1 and (7.1), we have, as $n \rightarrow \infty$ in this situation,

$$
q_{m}(n) \sim\left(2 \pi B^{2}\right)^{-1 / 2} \frac{\pi_{1}}{\pi_{2}},
$$

where

$$
\begin{aligned}
& \pi_{1}=\exp \left\{\sum_{j=1}^{n}\left(\frac{\sigma j}{1+e^{\sigma j}}+\log \left(1+e^{-\sigma j}\right)\right)\right\}, \\
& \pi_{2}=2^{m-1} \exp \left\{\sum_{j=1}^{m-1}\left(\frac{\sigma j}{1+e^{\sigma j}}+\log \left(\frac{1+e^{-\sigma j}}{2}\right)\right)\right\} .
\end{aligned}
$$

By (2.6) and (2.7), we also have

$$
\begin{gathered}
B^{2}=T_{0}-\sum_{j=1}^{m-1} \frac{j^{2} e^{\sigma j}}{\left(1+e^{\sigma j}\right)^{2}}=T_{0}+O\left(m^{3}\right), \\
n=S_{0}-\sum_{j=1}^{m-1} \frac{j}{1+e^{\sigma j}}=S_{0}+O\left(m^{2}\right),
\end{gathered}
$$

where

$$
S_{0}=\sum_{j=1}^{n} \frac{j}{1+e^{\sigma j}}, \quad T_{0}=\sum_{j=1}^{n} \frac{j^{2} e^{\sigma j}}{\left(1+e^{\sigma j}\right)^{2}} .
$$

We note that $\sigma S_{0}$ and $\sigma^{2} T_{0}$ were the sums considered in parts (ii) and (iii) of Lemma 12. For $m$ small compared with $n^{1 / 2}$ it follows from the above and Lemma 12 that

$$
\begin{aligned}
& \pi_{1}=\exp \left\{\frac{\pi^{2}}{6 \sigma}-\frac{1}{2} \log 2+O\left(\sigma(\log n)^{3}\right)\right\}, \quad T_{0} \sim \frac{\pi^{2}}{6 \sigma^{3}}, \\
& S_{0}=\frac{\pi^{2}}{12 \sigma^{2}}+O\left((\log n)^{3}\right) \sim \frac{\pi^{2}}{12 \sigma^{2}} .
\end{aligned}
$$


If $m=o\left(n^{1 / 3}\right)$, then $m^{3}=o(n)$, and $m^{2}=o(n)$ and since $T_{0}>S_{0} \geq n$ it then follows from (9.2) and (9.1) that

$$
S_{0} \sim n, \quad B^{2} \sim T_{0} \sim \frac{4.3^{1 / 2}}{\pi} n^{3 / 2},
$$

and so in order to estimate $q_{m}(n)$ in this case we only need to deal with $\pi_{1}$ and $\pi_{2}$. The result obtained will be as follows.

THEOREM 3. As $n \rightarrow \infty$, we have

$$
q_{m}(n) \sim \frac{1}{2^{m+1} 3^{1 / 4} n^{3 / 4}} \exp \left\{\pi\left(\left(\frac{n}{3}\right)^{1 / 2}+\frac{(m-1) m}{8}\left(\frac{1}{3 n}\right)^{1 / 2}\right)\right\}
$$

uniformly with respect to $m$ such that $m \geq 1, m=o\left(n^{1 / 3}\right)$. (More precisely, let $\left(\theta_{n}\right)$ be a decreasing sequence of positive numbers such that $\lim _{n \rightarrow \infty} \theta_{n}=0$. Then (9.6) holds uniformly with respect to $m$ such that

$$
1 \leq m \leq n^{1 / 3} \theta_{n},
$$

with all implied constants effective and independent of $m$ and $n$ (but dependent on the sequence $\left.\left(\theta_{n}\right)\right)$.)

PROOF. We assume that (9.7) holds and that $n \geq n_{1}$, for suitable $n_{1}$. In order to estimate $\sigma^{-1}$ more carefully and use (9.3) we consider the sum of the first $m-1$ terms of $S_{0}$. Since $\sigma \leq 2 n^{-1 / 2}$ and $m=o\left(n^{1 / 3}\right)$, for $j \leq m-1$ we have

$$
\frac{j}{1+e^{\sigma j}}=\frac{j}{2\left(1+\frac{1}{2} \sigma j+O\left((\sigma j)^{2}\right)\right)}=\frac{j}{2}+O\left(\sigma j^{2}\right),
$$

and hence

$$
\sum_{j=1}^{m-1} \frac{j}{1+e^{\sigma j}}=\frac{(m-1) m}{4}+O\left(\sigma m^{3}\right) .
$$

From (9.4) we have

$$
\begin{aligned}
\frac{1}{\sigma} & =\left\{\frac{12}{\pi^{2}} S_{0}+O\left((\log n)^{3}\right)\right\}^{1 / 2} \\
& =\left\{\frac{12}{\pi^{2}}\left(n+\frac{1}{4}(m-1) m+O\left(\frac{m^{3}}{n^{1 / 2}}+(\log n)^{3}\right)\right)\right\}^{1 / 2} \\
& =\frac{2.3^{1 / 2} n^{1 / 2}}{\pi}\left\{1+\frac{1}{4} \frac{(m-1) m}{n}+O\left(\frac{m^{3}}{n^{3 / 2}}+\frac{(\log n)^{3}}{n}\right)\right\}^{1 / 2} \\
& =\frac{2.3^{1 / 2} n^{1 / 2}}{\pi}\left\{1+\frac{1}{8} \frac{(m-1) m}{n}+O\left(\frac{m^{4}}{n^{2}}+\frac{m^{3}}{n^{3 / 2}}+\frac{(\log n)^{3}}{n}\right)\right\},
\end{aligned}
$$


that is,

$$
\frac{1}{\sigma}=\frac{2.3^{1 / 2} n^{1 / 2}}{\pi}+\frac{3^{1 / 2}}{4 \pi} \frac{(m-1) m}{n^{1 / 2}}+O\left(\frac{m^{3}}{n}+\frac{(\log n)^{3}}{n^{1 / 2}}\right) .
$$

Since the error term tends to zero as $n \rightarrow \infty$ it follows from (9.3) that as $n \rightarrow \infty$

$$
\pi_{1} \sim \exp \left\{\frac{\pi^{2}}{6}\left(\frac{2.3^{1 / 2} n^{1 / 2}}{\pi}+\frac{3^{1 / 2}(m-1) n}{4 \pi n^{1 / 2}}\right)-\frac{1}{2} \log 2\right\}
$$

Finally, we need to estimate $\pi_{2}$. We have

$$
\pi_{2}=2^{m-1} \exp \left\{\sum_{j=1}^{m-1} f(j)\right\}
$$

where for $j \leq m-1$

$$
\begin{aligned}
f(j) & =\frac{\sigma j}{1+e^{-\sigma j}}+\log \left(\frac{1+e^{-\sigma j}}{2}\right) \\
& =\frac{1}{2} \sigma j+O\left(\sigma^{2} j^{2}\right)-\frac{1}{2} \sigma j+O\left(\sigma^{2} j^{2}\right) \\
& =O\left(\sigma^{2} j^{2}\right) .
\end{aligned}
$$

Thus

$$
\sum_{j=1}^{m-1} f(j)=O\left(\sigma^{2} m^{3}\right)=O\left(m^{3} / n\right)
$$

and

$$
\pi_{2}=2^{m-1} \exp \left(O\left(m^{3} / n\right)\right),
$$

so that, by (9.7), as $n \rightarrow \infty, \pi_{2} \sim 2^{m-1}$. Combining this with our asymptotic estimates of $\pi_{1}$ and $B^{2}$ as in (9.8) and (9.5) gives the required result.

COROllary. As $n \rightarrow \infty$, we have

$$
q_{m}(n) \sim \frac{1}{2^{m+1} 3^{1 / 4} n^{3 / 4}} \exp \left\{\pi\left(\frac{n}{3}\right)^{1 / 2}\right\}
$$

uniformly with respect to $m$ such that $m \geq 1, m=o\left(n^{1 / 4}\right)$. In particular, taking $m=1$,

$$
q(n)=q_{1}(n) \sim \frac{1}{2^{2} 3^{1 / 4} n^{3 / 4}} \exp \left\{\pi\left(\frac{n}{3}\right)^{1 / 2}\right\},
$$

and for $m$ as above we have

$$
q_{m}(n) \sim \frac{1}{2^{m-1}} q_{1}(n)
$$


The result (9.9) was proved by Erdös and Szalay [4] for $m=o\left(n^{1 / 5}\right)$. Erdös, Nicolos and Szalay [3] obtained asymptotic relationships of this type connecting $q_{m}(n)$ and values of $q(n)$ for a wider range of values of $m$. Their results showed, in particular, that (9.9) holds for $m=o\left(n^{1 / 4}\right)$.

\section{References}

[1] L. Breiman, Probability (Addison-Wesley, Reading, 1968).

[2] J. Dixmier and J.-L. Nicolas, 'Partitions sans petits sommants', in: A Tribute to Paul Erdös (eds. A. Baker, B. Bollobás and A. Hajmal) (Cambridge University Press, Cambridge, 1990) pp. 121-152.

[3] P. Erdös, J.-L. Nicolas and M. Szalay, 'Partitions into parts which are unequal and large', preprint, Inst. Hautes Études Sci. Publ. Math., 1990.

[4] P. Erdös and M. Szalay, 'On the statistical theory of partitions', in: Topics in classical number theory, Coll. Math. Soc. Janos Bolyai 34 (North-Holland/Elsevier, Budapest, 1981) pp. 397-450.

[5] G. Freiman, 'Waring's problem with an increasing number of terms', Elabuz. Gos. Ped. Inst. Učen. Zap. 3 (1958), 105-119 (in Russian).

[6] - 'An analytical method of analysis of linear Boolean equations', in: Third Int. Conf. on Collective Phenomena (Moscow, 1978), Ann. New York Acad. Sci. 337 (New York Acad. Sci., New York, 1980) pp. 97-102.

[7] —-, 'On extremal additive problems of Paul Erdös', in: Proc. of Second Int. Conf. on Combinatorial Mathematics and Computing, Canberra, 1987, Ars Combin. 26B (1988) pp. 93-114.

[8] G. H. Hardy and S. Ramanujan, 'Asymptotic formulae in combinatory analysis', Proc. London Math. Soc. (2) 17 (1918), 75-1 15 (Also in Collected Papers of S. Ramanujan, (Cambridge Univ. Press, Cambridge, 1927), (Chelsea, New York, 1962) pp. 276-309).

[9] L. K. Hua, 'On the number of partitions of a number into unequal parts', Trans. Amer. Math. Soc. 51 (1942), 194-201.

[10] A. G. Postnikov, Vvedenie v analitičeskuiu teoriu čisel (in Russian), translated as Introduction to analytic number theory, Translations of Mathematical Monographs 68 (Amer. Math. Soc., Providence, 1987).

[11] K. Roth and G. Szekeres, 'Some asymptotic formulae in the theory of partitions', Quart. J. Math. Oxford Ser. (2) 5 (1954), 241-259.

School of Mathematical Sciences

Raymond and Beverly Sacler Faculty

of Exact Sciences

Tel-Aviv University

Ramat-Aviv, Tel-Aviv

Israel
Department of Pure Mathematics

University of Adelaide

S.A. 5005

Australia 